\author{
ANNALS OF “DUNAREA DE JOS” UNIVERSITY OF GALATI \\ MATHEMATICS, PHYSICS, THEORETICAL MECHANICS \\ FASCICLE II, YEAR X (XLI) 2018, No. 2
}

Article DOI: https://doi.org/10.35219/ann-ugal-math-phys-mec.2018.2.05

\title{
EXTENSION OF NOETHER'S THEOREM FOR THE RHEONOMIC LAGRANGIAN OF SECOND ORDER
}

Camelia Frigioiu

\author{
"Dunărea de Jos" University of Galati, Faculty of Science and Environment, \\ Department of Mathematics and Computer Science, 111 Domneasca Street, Galati, Romania \\ e-mail: cfrigioiu@ugal.ro
}

\begin{abstract}
This paper extends the Noether's theorem for the rheonomic Lagrangian of second order.
\end{abstract}

Keywords: rheonomic, Lagrange space, simmetry, action integral

\section{INTRODUCTION}

Time dependent Lagrangians are involved in many problems in Physics and Variational calculus. An explicit dependence on time in problems of dynamics has been considered by A. Wundheiler in paper [4] and he called the geometrical background related to it "Rheonomic Geometry". An extension of Lagrange geometry, i.e. that of the rheonomic Lagrange spaces was studied by M. Anastasiei in [1].

Noether's theorem is a property of any system that can be derived from an action and possesses some continuous symmetry. In words, to any given symmetry, Noether's algorithm associates a conserved charge to it.

In this paper we'll study different symmetries of a rheonomic Lagrange space of second order and their relations with conservation laws.

\section{MAIN RESULTS}

Let $\mathrm{M}$ be a $\mathrm{C}^{\infty}$ manifold of dimension $\mathrm{n}$ and the bundle $\left(O s c^{2} M, \pi, M\right)$. [3] A point $\mathrm{x} \in M$ has the local coordinates denoted with $\left(x^{i}\right), i=\overline{1, n}$ and the local coordinates of $u \in O s c^{2} M$ are $\left(x^{i}, y^{(1) i}, y^{(2) i}\right)$. The bundle $O s c^{2} M$ can be identified with the bundle of accelerations of second order of $M$, denoted with $T^{2} M$. [3]

The differentiable bundle

$$
E=\left(T^{2} M \times \boldsymbol{R}, \pi, M\right)
$$

is determined by the differential manifold $T^{2} M \times \boldsymbol{R}$ and the canonical projection $\pi: T^{2} M \times \boldsymbol{R} \rightarrow M$

$$
\pi\left(x, y^{(1)}, y^{(2)}, t\right)=x, \quad \forall\left(x, y^{(1)}, y^{(2)}, t\right) \in T^{2} M \times R .
$$

A rheonomic Lagrangian of second order is a mapping $L: E=T^{2} M \times \boldsymbol{R} \rightarrow \boldsymbol{R}$

$$
\left(x, y^{(1)}, y^{(2)}, t\right) \rightarrow L\left(x, y^{(1)}, y^{(2)}, t\right) \in \boldsymbol{R} .
$$

The Lagrangian $L$ is called differentiable if it is of class $C^{\infty}$ on $E=T^{2} M \times R$ and continuous in the points $(x, 0,0, t) \in T^{2} M \times R$. 
A symmetry for the rheonomic differential Lagrangian $L\left(x, y^{(1)}, y^{(2)}, t\right)$ is a $\mathrm{C}^{\infty}$ diffeomorphism $\varphi: M \times \boldsymbol{R} \rightarrow M \times \boldsymbol{R}$, which preserves the variational principle for the action integral

$$
I(c)=\int_{0}^{1} L\left(x, \frac{d x}{d t}, \frac{1}{2} \frac{d^{2} x}{d t^{2}}, t\right) d t
$$

We will consider a local symmetry for the rheonomic Lagrangian of second order $L$, using the local diffeomorphism $\varphi$ defined on an open subset $U \times(a, b) \subset M \times \boldsymbol{R}$.

Now we study the infinitesimal symmetries on the open subset $U \times(a, b) \subset M \times \boldsymbol{R}$, defined by

$$
\begin{aligned}
x^{i} & =x^{i}+\varepsilon V^{i}(x, t) \quad(i=1, \ldots, n) \\
t^{\prime} & =t+\varepsilon \tau(x, t)
\end{aligned}
$$

where $\varepsilon$ is a real number, sufficiently small in absolute value so that the points $(x, t)$ and $\left(x^{\prime}, t^{\prime}\right)$ belong to the same domain of a local map $U \times(a, b)$ where the parametrized curve $c: t \in[0,1] \rightarrow\left(x^{i}(t), t\right) \in U \times(a, b)$ is defined. $V^{i}(x, t)$ is a vectorial field on the open set $U \times(a, b)$.

The inverse of the local diffeomorphism (2) is given by:

$$
\begin{aligned}
x^{i} & =x^{i}-\varepsilon V^{i}(x, t) \\
t & =t^{\prime}-\varepsilon \tau(x, t) .
\end{aligned}
$$

Vectorial field $V^{i}(x(t), t)=V^{i}(t)$ satisfies in the endpoints $c(0)$ and $c(1)$ the following equations

$$
V^{i}(0)=V^{i}(1)=0 ; \quad \frac{d V^{i}}{d t}(0)=\frac{d V^{i}}{d t}(1)=0 .
$$

An infinitesimal transformation (2) is a symmetry for Lagrangian $L\left(x, y^{(1)}, y^{(2)}, t\right)$ if and only if for any function $C^{\infty}$ differentiable. $\Phi\left(\mathrm{x}, y^{(1)}, t\right)$ the following equality holds

$$
L\left(x^{\prime}(t), \frac{d x^{\prime}}{d t^{\prime}}, \frac{1}{2} \frac{d^{2} x^{\prime}}{d t^{\prime 2}}, t^{\prime}\right) d t^{\prime}=\left\{L\left(x, \frac{d x}{d t}, \frac{1}{2} \frac{d^{2} x}{d t^{2}}, t\right)+\Phi\left(x, \frac{d x}{d t}, t\right)\right\} d t .
$$

From (2) one obtains

$$
\frac{d t^{\prime}}{d t}=1+\varepsilon \frac{d \tau}{d t} ; \frac{d x^{i}}{d t^{\prime}}=\frac{d x^{i}}{d t}+\varepsilon \varphi^{(1) \mathrm{i}} ; \frac{1}{2} \frac{d^{2} x^{i}}{d t^{\prime 2}}=\frac{1}{2}\left[\frac{d^{2} x^{i}}{d t^{2}}+\varepsilon \varphi^{(2) i}\right]
$$

where

$$
\varphi^{(1) \mathrm{i}}=\frac{d V^{i}}{d t}-\frac{d x^{i}}{d t} \frac{d \tau}{d t} ; \quad \varphi^{(2) \mathrm{i}}=\frac{d^{2} V^{i}}{d t^{2}}-2 \frac{d^{2} x^{i}}{d t^{2}} \frac{d \tau}{d t}-\frac{d x^{i}}{d t} \frac{d^{2} \tau}{d t^{2}}
$$

If we substitute (5) and (6) into (4) and we neglect the terms in $\varepsilon, \varepsilon^{2}, \ldots$, we replace $\Phi$ with $\varepsilon \Phi$, one obtains

$$
\frac{\partial L}{\partial t} \tau+\frac{\partial L}{\partial x^{i}} \mathrm{~V}^{\mathrm{i}}+\frac{\partial L}{\partial y^{(1) i}} \varphi^{(1) \mathrm{i}}+\frac{1}{2} \frac{\partial L}{\partial y^{(2) i}} \varphi^{(2) \mathrm{i}}+\mathrm{L} \frac{d \tau}{d t}=\frac{d \Phi}{d t} .
$$

Using scalar fields

$$
\stackrel{1}{I}(L)=y^{(1) i} \frac{\partial L}{\partial y^{(2) i}} ; \quad \stackrel{2}{I}(L)=y^{(1) i} \frac{\partial L}{\partial y^{(1) i}}+2 y^{(2) i} \frac{\partial L}{\partial y^{(2) i}} .
$$

equation (7) can be written under the following form

$$
\mathrm{V}^{\mathrm{i}} \frac{\partial L}{\partial x^{i}}+\frac{d V^{i}}{d t} \frac{\partial L}{\partial y^{(1) i}}+\frac{1}{2} \frac{d^{2} V^{i}}{d t^{2}} \frac{\partial L}{\partial y^{(2) i}}+\left\{L \frac{d \tau}{d t}+\tau \frac{\partial L}{\partial t}-\left[2(L) \frac{d \tau}{d t}+\frac{1}{2} I(L) \frac{d^{2} \tau}{d t^{2}}\right]\right\}=\frac{d \Phi}{d t} .
$$


Using the operator $\frac{d_{V}}{d t}=V^{i} \frac{\partial}{\partial x^{i}}+\frac{d V^{i}}{d t} \frac{\partial}{\partial y^{(1) i}}+\frac{1}{2} \frac{d^{2} V^{i}}{d t^{2}} \frac{\partial}{\partial y^{(2) i}}$ we have the theorem:

Theorem 1. A necessary and sufficient condition for infinitesimal transformation (2) to be a symmetry for the rheonomic Lagrangian $L\left(x, y^{(1)}, y^{(2)}, t\right)$ is the left-hand side of equality

$$
\left.\frac{d_{v} L}{d t}+\left\{L \frac{d \tau}{d t}+\tau \frac{\partial L}{\partial t}-[2 / L) \frac{d \tau}{d t}+\frac{1}{2} I(L) \frac{d^{2} \tau}{d t^{2}}\right]\right\}=\frac{d \Phi}{d t}
$$

be equal to $\frac{d}{d t} \Phi\left(x, y^{(1)}, t\right)$ on the smooth curve $c$.

A straightforward calculation leads to following theorem.

Theorem 2. Along any smooth curve $c$ from the manifold $M$, for any rheonomic Lagrangian of second order the following relation holds:

$$
\frac{d_{V} L}{d t}=\stackrel{\circ}{E}_{i}(L) V^{i}+\frac{d}{d t}\left(\stackrel{2}{I} I_{V}(L)\right)-\frac{1}{2} \frac{d^{2}}{d t^{2}}\left(I_{V}^{1}(L)\right),
$$

where $\stackrel{\circ}{E}_{i}(L)=\frac{\partial L}{\partial x^{i}}-\frac{d}{d t} \frac{\partial L}{\partial y^{(1) i}}+\frac{1}{2} \frac{d^{2}}{d t^{2}}\left(\frac{\partial L}{\partial y^{(2) i}}\right)$.

Along the curve $\mathrm{c}$, the function defined by

$$
\varepsilon_{c}(\mathrm{~L})=-\frac{1}{2} \stackrel{1}{I}(\mathrm{~L})
$$

is called the energy of first order for the Lagrangian $L\left(x, y^{(1)}, y^{(2)}, t\right)$,

and the function defined by

$$
\stackrel{2}{E}_{c}(L)=\stackrel{2}{I}(L)-\frac{1}{2} \frac{d}{d t} \stackrel{1}{I}(L)-L\left(x, y^{(1)}, y^{(2)}, t\right)
$$

is named the energy of second order for Lagrangian $L\left(x, y^{(1)}, y^{(2)}, t\right)$.

Theorem 3. For every smooth curve $\mathrm{c}$ on the base manifold $M$ the following formula holds

$$
\frac{d \stackrel{1}{\varepsilon}(L)}{d t}+\frac{1}{2} \stackrel{2}{I}(\mathrm{~L})=-\frac{1}{2} \frac{d x^{i}}{d t} \stackrel{1}{E}(\mathrm{~L}),
$$

with $\stackrel{1}{E}_{i}=-\frac{\partial}{\partial y^{(1) i}}+\frac{d}{d t} \frac{\partial}{\partial y^{(2) i}}$.

According Theorem 2 and Theorem 3, equation (9) can be written in the following form

$$
\begin{aligned}
& \mathrm{V}^{\mathrm{i}} \stackrel{o}{E}_{i}(\mathrm{~L})+\tau \frac{\partial L}{\partial t}+\frac{d}{d t} \stackrel{2}{I} \mathrm{v}(\mathrm{L})-\frac{1}{2} \frac{d^{2}}{d t^{2}} \stackrel{1}{I}_{\mathrm{v}}(\mathrm{L})+\tau \frac{d}{d t} \stackrel{2}{\varepsilon_{c}}(\mathrm{~L})+\frac{d}{d t}\left[-\tau{ }^{2} \varepsilon_{c}(L)+\frac{d \tau}{d t} \stackrel{1}{\varepsilon_{c}}(L)\right]=\frac{d \Phi}{d t} \Leftrightarrow \\
& V^{i} \stackrel{o}{E}_{i}(L)+\tau\left(\frac{\partial L}{\partial t}+\frac{d \varepsilon_{c}^{2}}{d t}\right)+\frac{d}{d t}\left(\stackrel{2}{I_{V}}(L)-\frac{1}{2} \frac{d}{d t} I_{V}^{1}(L)-\tau \stackrel{2}{\varepsilon_{c}}(L)+\varepsilon_{c}^{1}(L) \frac{d \tau}{d t}-\Phi\right)=0 .
\end{aligned}
$$

Using the above equation we can write a theorem of type Noether

Theorem 4. For any infinitesimal symmetry (2) (which verify (9)) of a rheonomic Lagrangian $L\left(x, y^{(1)}, y^{(2)}, t\right)$ and for any $C^{\infty}$ function, $\Phi\left(x, y^{(1)}, t\right)$, the following function 


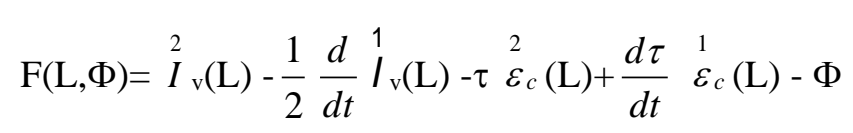

is conserved on the solution curves of the Euler-Lagrange equations

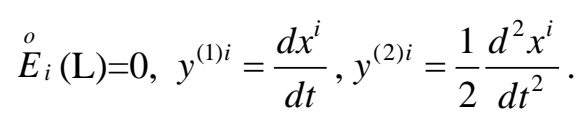

Especially if the Zermelo conditions are checked this results lead us to obtain a new theorem of type Noether.

Theorem 5. For any infinitesimal symmetry (2) of a rheonomic Lagrangian $L\left(x, y^{(1)}, y^{(2)}, t\right)$, which verify the Zermelo equations

$$
\stackrel{1}{I}_{v}(L)=0, \stackrel{2}{I}_{v}(L)=L
$$

and for any $C^{\infty}$ function $\Phi\left(x, y^{(1)}, t\right)$, the following function

$$
F(L, \Phi)=\stackrel{2}{I}_{v}(L)-\frac{1}{2} \frac{d}{d t} \stackrel{1}{I}_{v}(L)-\Phi
$$

is conserved along the solution curves of the Euler-Lagrange equations ${ }_{i}^{o}(\mathrm{~L})=0, \quad y^{(1) i}=\frac{d x^{i}}{d t}$, $y^{(2) i}=\frac{1}{2} \frac{d^{2} x^{i}}{d t^{2}}$.

\section{References}

1. M. Anastasiei, On the geometry of time-dependent Lagrangians, Mathematical and Computing Modelling, 20, no4/5, Pergamon Press, 1994.

2. M.de Leon, P.R.Rodriguez, Methods of Differential Geometry in Analitical Mechanics. NorthHolland,1989.

3. R. Miron, The Geometry of Higher-Order Lagrange Spaces. Applications to Mechanics and Physics, Kluwer Academic Publishers, FTPH, no. 82, 1997

4. A. Wundheiler, Rheonome Geometrie. Absolute Mechanik, Prace Matematyczno -Fizyczne, 40 , issue $1,1933,97-142$ 